\title{
Multidrug-Resistant Shigellosis among Children Aged below Five Years with Diarrhea at Banadir Hospital in Mogadishu, Somalia
}

\author{
Bilan Sheikh Ali Nor $\mathbb{D}^{1},{ }^{1,2,3}$ Nelson Chengo Menza ${ }^{(D)},{ }^{1}$ and Abednego Moki Musyoki ${ }^{1}{ }^{1}$ \\ ${ }^{1}$ Department of Medical Laboratory Sciences, Kenyatta University, Nairobi, Kenya \\ ${ }^{2}$ Department of Pediatrics, Banadir Hospital, Mogadishu, Somalia \\ ${ }^{3}$ National Public Health Reference Laboratory (NPHRL) in Mogadishu, Mogadishu, Somalia
}

Correspondence should be addressed to Bilan Sheikh Ali Nor; bilansheikhali121@gmail.com

Received 13 April 2021; Accepted 29 May 2021; Published 9 June 2021

Academic Editor: Jorge Garbino

Copyright (C) 2021 Bilan Sheikh Ali Nor et al. This is an open access article distributed under the Creative Commons Attribution License, which permits unrestricted use, distribution, and reproduction in any medium, provided the original work is properly cited.

\begin{abstract}
Globally, shigellosis remains the second leading cause of diarrhea-associated deaths among children under five years of age, and the infections are disproportionately higher in resource-limited settings due to overcrowding, poor sanitation, and inadequate safe drinking water. The emergence and global spread of multidrug-resistant (MDR) Shigella are exacerbating the shigellosis burden. We adopted a cross-sectional study design to determine the distribution and antimicrobial susceptibility (AST) patterns of Shigella serogroups among children aged below five years presenting with diarrhea at Banadir Hospital in Mogadishu, Somalia, from August to October 2019. Stool and rectal swab samples were collected from 180 children consecutively enrolled using a convenient sampling technique and processed following standard bacteriological methods. AST was determined using the Kirby-Bauer disc diffusion method and interpreted as per the Clinical Laboratory Standard Institute (2018) guidelines. Shigellosis prevalence was $20.6 \%(37 / 180)$, and S. flexneri $(26 / 37(70.3 \%)$ ) was the predominant serogroup. All the serogroups were $100 \%$ resistant to ampicillin (AMP), trimethoprim-sulfamethoxazole (SXT), and tetracycline (TE). Ceftriaxone (CRO) resistance was the highest among S. sonnei (66.7\%) isolates. 19.2\% of S. flexneri and S. sonnei (50\%) serogroups were resistant to ciprofloxacin (CIP), but all S. dysenteriae type 1 isolates remained (100\%) susceptible. Forty percent of CIP-susceptible $S$. dysenteriae type 1 were resistant to CRO. Seven MDR Shigella phenotypes were identified, dominated by those involving resistance to AMP, SXT, and TE (100\%). Our findings showed a high prevalence of shigellosis with $S$. flexneri as the most predominant serogroup among children under five years of age in Banadir Hospital, Somalia. AMP and SXT are no longer appropriate treatments for shigellosis in children under five years in Banadir Hospital. MDR Shigella strains, including those resistant to CIP and CRO, have emerged in Somalia, posing a public health challenge. Therefore, there is an urgent need for AMR surveillance and continuous monitoring to mitigate the further spread of the MDR Shigella strains in Banadir Hospital and beyond.
\end{abstract}

\section{Introduction}

Globally, approximately 165 million episodes of bacillary dysentery (shigellosis) and about 1.1 million associated deaths occur annually [1]. Developing countries, whereby $69 \%$ of the episodes and $61 \%$ of deaths occur among children under five years, bear the highest burden of these infections (99\%) [2]. This disproportionate shigellosis burden is attributable to overcrowding, poor sanitation, and limited access to safe drinking water $[2,3]$. The causative agent of bacillary dysentery is Shigella bacteria. They are facultative anaerobes, nonmotile, and none spore-forming Gramnegative microorganisms. Serologically the pathogen is grouped into four distinct serogroups, namely Shigella dysenteriae (Shiga bacillus), S. flexneri, S. boydii, and $S$. sonnei. The distribution of these serogroups varies geographically, whereby S. flexneri and S. sonnei are predominant in developing and developed countries, respectively [1].

Even though most cases of dysentery are usually selflimiting, appropriate antimicrobial therapy prescription is required to improve the clinical outcome of the disease, 
decrease the period of illness, and reduce bacterial shedding time [4]. Ciprofloxacin is the World Health Organization (WHO) first-line treatment option for dysentery. Pivmecillinam, ceftriaxone, or azithromycin (in adults only) are the second-line treatment options [2]. However, Shigella strains resistant to these antibiotics are increasingly emerging and spreading globally, thus posing a serious public health threat [5].

In Somalia, three-quarters of the population lacks sanitary facilities, $45 \%$ have no access to clean and safe drinking water, and about 3.2 million lack access to healthcare facilities [6,7]. Currently, the country is facing a public health crisis due to civil war and political unrest that have been ongoing for decades affecting the establishment of functional healthcare service delivery systems. Also, the poorly managed and overcrowded internally displaced persons (IDP) camps with no access to adequate safe drinking water and sanitary facilities serve as hotspots for diarrhea disease outbreaks. As per the findings of a study carried out by Global Burden of Disease (1990 to 2015), Somalia had the highest diarrhea mortality among children under five years in Eastern Mediterranean Region, and shigellosis was the second topmost killer [6]. Unpublished data in Somalia show that the traditional first-line empiric therapy antibiotics, including co-trimoxazole and ampicillin, are used in shigellosis treatment, against the WHO guidelines. This poses a serious public health concern due to multidrugresistant infections. Yet, current epidemiological data on bacillary dysentery in Somalia are limited. Scientifically based information is critical for defining an appropriate empiric therapy and preventing the emergence and spread of multidrug-resistant shigellosis in Somalia. Therefore, this study aimed to determine the distribution and antimicrobial susceptibility patterns of Shigella serogroups among children of less than five years presenting with diarrhea at Banadir Hospital in Mogadishu, Somalia.

\section{Methods}

2.1. Design. A cross-sectional study to determine the distribution and antibiotic susceptibility of Shigella serogroups among children under five years was carried out at Banadir Hospital from August to October 2019.

2.2. Setting and Sample Collection. Banadir Hospital is located in Wadajir District, South West of the Banadir region of Somalia. It is the largest mother and child hospital (MCH) in Mogadishu, Somalia. The study excluded children with a history of antibiotic use within two weeks before the sample collection date. A structured questionnaire was completed for each participant through a face-to-face interview to assess the factors associated with shigellosis. A total of 180 samples, including 34 stool and 146 rectal swab samples, were collected from children with clinically diagnosed diarrhea. We collected stool samples in sterile screw-cap containers with Cary Blair Transport Medium (Medical Chemical Corporation, Torrance, CA, USA) [8]. In cases where stool samples were not available, rectal swabs were collected and immediately placed in the Cary Blair Transport Medium. The samples were then transported in an icebox to the National Public Health Reference Laboratory (NPHRL) in Mogadishu, Somalia, for analysis within 2-3 hours.

2.3. Bacterial Isolation, Biochemical Characterization, and Serogrouping. We isolated and identified Shigella as per the standard bacteriological methods $[9,10]$. Samples were aseptically inoculated into selenite F broth (Oxoid, UK) and incubated aerobically at $37^{\circ} \mathrm{C}$ for $18-24$ hours; subsequently, a loopful of the culture was streaked on MacConkey agar (Merck, Germany) and xylose lysine deoxycholate (XLD) agar (Oxoid, UK) and incubated aerobically at $37^{\circ} \mathrm{C}$ for 18-24 hours. Biochemical tests including carbohydrates fermentation (triple sugar iron agar), urea test, indole test, and motility test were then used to identify the resultant colonies. Shigella serogroups were determined using a commercially available serological kit (Denka Seiken, Tokyo, Japan).

2.4. Antimicrobial Susceptibility Testing. The choice of the antibiotics tested in this study was guided by clinical data on drugs used in the management of diarrheal diseases in Banadir Hospital, Somalia. To this end, we tested the antimicrobial susceptibility of Shigella serogroups to six antibiotics: including ampicillin $(10 \mu \mathrm{g})$, tetracycline $(30 \mu \mathrm{g})$, trimethoprim-sulfamethoxazole $(1.25 \mu \mathrm{g})$, ciprofloxacin $(30 \mu \mathrm{g})$, ceftriaxone $(30 \mu \mathrm{g})$, and azithromycin $(15 \mu \mathrm{g})$ (Oxoid, UK) using the Kirby-Bauer disc diffusion method. A bacterium suspension equivalent to 0.5 McFarland standard was prepared and inoculated on Mueller-Hinton agar (Merck, Germany). The plates were allowed to dry for 3-5 minutes, antimicrobial discs were placed on the medium, and subsequently, the culture plates were incubated at $37^{\circ} \mathrm{C}$ for 24 hours. The minimum inhibition zones were determined and interpreted following the Clinical Laboratory Standards Institute (CLSI, 2018) [11] guidelines, and Escherichia coli strain (ATCC 25922) was used as a quality control organism.

2.5. Data Analysis. The obtained data were analyzed using IBM SPSS version 23 software. Descriptive data were generated and presented in tables, and the Chi-square $\left(X^{2}\right)$ statistic was used to test the relationship between the occurrence of shigellosis and the patient's sociodemographic characteristics and environmental conditions. Variables showing a significant relationship using $X^{2}$ were further analyzed using Pearson correlation statistics. A $p$ value less than 0.05 was considered statistically significant.

\section{Results}

3.1. Sociodemographic Characteristics of Children and Caregivers. We sampled 180 children under the age of five presenting with diarrhea at Banadir Hospital, Somalia. Of these, 69/180 (38.3\%) and 111/180 (61.7\%) were male and female, respectively. The mean age of the children was 20.1 
months (standard deviation of 12.87 months). Based on the age, we categorized the children into five groups, with the majority under the age group $>12-24$ months $(43.3 \%)$ and living in households with two children (48.9\%). Most of the caregivers aged between 25 and 30 years (41.1\%), were married (74.4\%), and had no formal education (78.4\%) (Table 1).

3.2. Prevalence of Shigellosis and Shigella Serogroups' Distribution among Children under Five. The overall prevalence of shigellosis among children presenting with diarrhea at Banadir Hospital was 20.6\% (37/180). We isolated three Shigella serogroups: serogroup A (Shigella dysenteriae type 1), B (S. flexneri), and C (S. sonnei). Serogroup D (S. boydii) was absent. Shigella flexneri $(26 / 37,70.3 \%)$ was the most predominant serogroup, followed by $S$. sonnei $(6 / 37,16.2 \%)$ and $S$. dysenteriae $(5 / 37,13.5 \%)(t=15.187 ; p=0.0001)$.

3.3. Antimicrobial Susceptibility Patterns of Shigella Serogroups. All the isolated Shigella serogroups were 100\% resistant to ampicillin, trimethoprim-sulfamethoxazole, and tetracycline (Table 2). Additionally, 19.2\% S. flexneri and S. sonnei (50\%) were resistant to ciprofloxacin, but S. dysenteriae type 1 remained susceptible. However, $40 \%$ of the ciprofloxacin-susceptible $S$. dysenteriae type 1 were ceftriaxone resistant. The highest resistance to ceftriaxone occurred among S. sonnei (66.7\%) serogroup, followed by S. dysenteriae type $1(40 \%)$ and S. flexneri (38.5\%). None of the serogroups was resistant to azithromycin (Table 2).

Based on resistance to two or more classes of antibiotics, we categorized the Shigella serogroups into seven multidrugresistant (MDR) phenotypes. The most dominant phenotype was $A M P / S X T / T E(37 / 37,100 \%)$, followed by $A M P / S X T /$ CRO or SXT/TE/CRO (6/37, 42.2\%), AMP/SXT/TE/CRO (13/37, 35.1\%), AMP/SXT/CIP or SXT/TE/CIP (8/37, 21.6\%), AMP/SXT/TE/CIP $(5 / 37,13.6 \%)$, and AMP/SXT/TE/CIP/ $C R O(3 / 37,8.1 \%)$. MDR phenotypes of $S$. dysenteriae type 1 (AMP/SXT or AMP/TE or SXT/TE, and AMP/SXT/TE) were susceptible to ciprofloxacin, but $50 \%(3 / 6)$ of $S$. sonnei isolates and S. flexneri $(19.5 \%, 5 / 26)$ remained resistant. Among the serogroups, most of the MDR phenotypes were found in S. flexneri (65.9\%), followed by S. sonnei (20.7\%) and $S$. dysenteriae type 1 (13.4\%) isolates.

3.4. Factors Associated with Shigellosis among Children under Five Years of Age. Even though Shigella infections appeared to be higher among children in the age group $>12-24$ months $(43.3 \%)(\chi 2=10.601, p$ value $=0.031)$ whose caregivers had no formal education $(\chi 2=13.515 ; p=0.004)$, correlation statistical analysis showed no such relationship (Table 3).

\section{Discussion}

We investigated the distribution and antibiotic susceptibility of Shigella among children under the age of five years in Somalia, a country with the highest burden of diarrhea
TABLE 1: Sociodemographic characteristics of children and caregivers.

\begin{tabular}{|c|c|c|}
\hline Category & Frequency & Percentage (\%) \\
\hline \multicolumn{3}{|l|}{ Gender } \\
\hline Male & 69 & 38.3 \\
\hline Female & 111 & 61.7 \\
\hline \multicolumn{3}{|l|}{ Age group of children (months) } \\
\hline $0-12$ & 67 & 37.2 \\
\hline$>12-24$ & 78 & 43.3 \\
\hline$>24-36$ & 19 & 10.6 \\
\hline$>36-48$ & 10 & 5.6 \\
\hline$>48-60$ & 6 & 3.3 \\
\hline \multicolumn{3}{|l|}{ Age group of caregivers } \\
\hline$<20$ & 11 & 6.1 \\
\hline$>20-25$ & 28 & 15.6 \\
\hline$>25-30$ & 74 & 41.1 \\
\hline$>30-35$ & 43 & 23.9 \\
\hline$>35-40$ & 24 & 13.3 \\
\hline \multicolumn{3}{|l|}{ Number of children per household } \\
\hline 1 & 61 & 33.9 \\
\hline 2 & 88 & 48.9 \\
\hline 3 & 29 & 16.1 \\
\hline 4 & 2 & 1.1 \\
\hline \multicolumn{3}{|l|}{ Marital status of the caregivers } \\
\hline Single & 2 & 1.1 \\
\hline Married & 134 & 74.4 \\
\hline Divorced & 26 & 14.4 \\
\hline Widowed/widower & 18 & 10 \\
\hline \multicolumn{3}{|l|}{ Education level of the caregivers } \\
\hline No formal education & 132 & 73.3 \\
\hline Primary school dropout & 37 & 20.6 \\
\hline Primary school & 8 & 4.4 \\
\hline Secondary school and above & 3 & 1.7 \\
\hline \multicolumn{3}{|l|}{ Number of people per household } \\
\hline $1-2$ & 6 & 3.3 \\
\hline $3-4$ & 58 & 32.2 \\
\hline $5-6$ & 72 & 40 \\
\hline $7-8$ & 39 & 21.7 \\
\hline $9-10$ & 4 & 2.2 \\
\hline $11-12$ & 1 & 0.5 \\
\hline
\end{tabular}

mortality among children less than five years in the Eastern Mediterranean Region [6]. The overall prevalence of shigellosis in Banadir Hospital was 20.6\%, higher than 4.0\%$17.8 \%$ documented in Kenya [12], Ethiopia [13, 14], Tanzania, South Africa, Pakistan, Nepal, Peru, Bangladesh, Brazil, and India [15]. This discrepancy suggests poor sanitary conditions, limited access to safe drinking water, and poor hygiene in Somalia. Moreover, the decades-long civil and political unrest in Somalia has negatively impacted healthcare service delivery and rehabilitation of drinking water and sewage systems. According to the United Nations Children's Fund (UNICEF), three-quarters of Somalia population lacks sanitary facilities, and $45 \%$ have no access to safe drinking water in Somalia [7-16].

Three Shigella serogroups, serogroup A (Shigella dysenteriae type 1), B (S. flexneri), and C (S. sonnei), caused bacillary dysentery among children under five years at Banadir Hospital, Somalia. Serogroup D (Shigella boydii) was absent, an unsurprising finding since this bacterium is more prevalent in Southeast Asia (Nepal and Bangladesh) 
TABLe 2: Antibiotic susceptibility pattern of Shigella serogroups isolated.

\begin{tabular}{|c|c|c|c|c|}
\hline \multirow{2}{*}{ Antibiotics } & \multirow{2}{*}{ Susceptibility } & \multicolumn{3}{|c|}{ Shigella serogroups } \\
\hline & & Shigella dysenteriae type $1, n(\%)$ & Shigella flexneri, $n(\%)$ & Shigella sonnei, $n(\%)$ \\
\hline \multirow{2}{*}{ AMP } & S & $0(0)$ & $0(0)$ & $0(0)$ \\
\hline & $\mathrm{R}$ & $5(100)$ & $26(100)$ & $6(100)$ \\
\hline \multirow{2}{*}{ CIP } & $\mathrm{S}$ & $5(100)$ & $21(80.8)$ & $3(50)$ \\
\hline & $\mathrm{R}$ & $0(0)$ & $5(19.2)$ & $3(50)$ \\
\hline \multirow{2}{*}{ SXT } & $\mathrm{S}$ & $0(0)$ & $0(0)$ & $0(0)$ \\
\hline & $\mathrm{R}$ & $5(100)$ & $26(100)$ & $6(100)$ \\
\hline \multirow{2}{*}{ CRO } & $\mathrm{S}$ & $3(60)$ & $16(61.5)$ & $2(33.3)$ \\
\hline & $\mathrm{R}$ & $2(40)$ & $10(38.5)$ & $4(66.7)$ \\
\hline \multirow{2}{*}{$\mathrm{TE}$} & $\mathrm{S}$ & $0(0)$ & $0(0)$ & $0(0)$ \\
\hline & $\mathrm{R}$ & $5(100)$ & $26(100)$ & $6(100)$ \\
\hline \multirow{2}{*}{$\mathrm{AZM}$} & S & $5(100)$ & $26(100)$ & $6(100)$ \\
\hline & $\mathrm{R}$ & $0(0)$ & $0(0)$ & $0(0)$ \\
\hline
\end{tabular}

AMP: ampicillin $(10 \mathrm{mg})$; TE: tetracycline $(30 \mathrm{mg})$; CRO: ceftriaxone $(30 \mathrm{mg})$; SXT: trimethoprim-sulfamethoxazole (co-trimoxazole) (25 mg); CIP: ciprofloxacin $(5 \mathrm{mg})$; AZM: azithromycin $(15 \mathrm{mg})$; S: susceptible; R: resistance.

TABLE 3: Risk factors for shigellosis among children under five years of age.

\begin{tabular}{|c|c|c|c|c|c|c|}
\hline \multirow{2}{*}{ Category } & \multicolumn{2}{|c|}{ Shigellosis } & \multirow{2}{*}{$\chi^{2}$ value } & \multirow{2}{*}{$p$ value } & \multirow{2}{*}{ COR $(95 \%)$} & \multirow{2}{*}{$p$ value } \\
\hline & Present, $n(\%)$ & Absent, $n(\%)$ & & & & \\
\hline \multicolumn{7}{|l|}{ Gender } \\
\hline Male & $10(5.6)$ & $59(41.3)$ & \multirow{2}{*}{2.519} & \multirow{2}{*}{0.113} & \multirow[b]{2}{*}{-} & \multirow[b]{2}{*}{-} \\
\hline Female & $27(15)$ & $84(58.7)$ & & & & \\
\hline \multicolumn{7}{|l|}{ Age group of children (months) } \\
\hline $0-12$ & $6(16.2)$ & $61(42.7)$ & \multirow{5}{*}{10.601} & \multirow{5}{*}{$0.031^{* *}$} & \multirow{5}{*}{0.138} & \multirow{5}{*}{0.064} \\
\hline$>12-24$ & $21(56.7)$ & $57(39.9)$ & & & & \\
\hline$>24-36$ & $7(18.9)$ & $12(8.4)$ & & & & \\
\hline$>36-48$ & $2(5.4)$ & $8(5.6)$ & & & & \\
\hline$>48-60$ & $1(2.7)$ & $5(3.5)$ & & & & \\
\hline \multicolumn{7}{|l|}{ Age group of caregivers } \\
\hline$<20$ & $1(2.7)$ & $10(7.5)$ & \multirow{5}{*}{4.477} & \multirow{5}{*}{0.345} & \multirow{5}{*}{-} & \multirow{5}{*}{-} \\
\hline$>20-25$ & $4(10.8)$ & $24(18.0)$ & & & & \\
\hline$>25-30$ & $13(35.1)$ & $51(38.3)$ & & & & \\
\hline$>30-35$ & $12(32.4)$ & $31(23.3)$ & & & & \\
\hline$>35-40$ & $7(18.9)$ & $17(12.8)$ & & & & \\
\hline \multicolumn{7}{|l|}{ Marital status of the caregivers } \\
\hline Single & $0(0)$ & $2(1.4)$ & \multirow{4}{*}{2.034} & \multirow{4}{*}{0.565} & \multirow{4}{*}{-} & \multirow{4}{*}{-} \\
\hline Married & $25(67.5)$ & $109(76.2)$ & & & & \\
\hline Divorced & $7(18.9)$ & $19(13.3)$ & & & & \\
\hline Widowed/widower & $5(13.5)$ & $13(9.1)$ & & & & \\
\hline Education level of the caregiver & & & & & & \\
\hline No formal education & $29(78.4)$ & $103(72.0)$ & & & & \\
\hline Primary school dropout & $2(5.4)$ & $35(24.5)$ & & & & \\
\hline Primary school & $4(10.8)$ & $4(2.8)$ & 13.515 & 0.004 & 0.058 & 0.439 \\
\hline Secondary school and above & $2(5.4)$ & $1(0.7)$ & & & & \\
\hline Number of children per househ & & & & & & \\
\hline 1 & $11(29.7)$ & $50(34.9)$ & & & & \\
\hline 2 & $20(54.1)$ & $68(47.6)$ & & & & \\
\hline 3 & $6(16.2)$ & $23(16.1)$ & 1.010 & 0.908 & - & - \\
\hline 4 & $0(0)$ & $2(1.4)$ & & & & \\
\hline Number of people per househo & & & & & & \\
\hline $1-2$ & $2(5.4)$ & $4(2.8)$ & & & & \\
\hline $3-4$ & $13(35.1)$ & $45(31.5)$ & & & & \\
\hline $5-6$ & $15(40.5)$ & $57(39.9)$ & & & & \\
\hline $7-8$ & $6(16.2)$ & $33(23.1)$ & 8.114 & 0.618 & - & - \\
\hline $9-10$ & $1(2.7)$ & $3(2.1)$ & & & & \\
\hline $11-12$ & $0(0)$ & $1(0.7)$ & & & & \\
\hline Sources of family income & & & & & & \\
\hline
\end{tabular}


TABle 3: Continued.

\begin{tabular}{|c|c|c|c|c|c|c|}
\hline \multirow{2}{*}{ Category } & \multicolumn{2}{|c|}{ Shigellosis } & \multirow{2}{*}{$\chi^{2}$ value } & \multirow{2}{*}{$p$ value } & \multirow{2}{*}{ COR $(95 \%)$} & \multirow{2}{*}{$p$ value } \\
\hline & Present, $n(\%)$ & Absent, $n(\%)$ & & & & \\
\hline Wages & $9(24.3)$ & $32(22.4)$ & \multirow{4}{*}{1.158} & \multirow{4}{*}{0.282} & \multirow{4}{*}{-} & \multirow{4}{*}{-} \\
\hline Own business & $12(32.4)$ & $65(45.5)$ & & & & \\
\hline Agriculture & $4(10.8)$ & $12(8.4)$ & & & & \\
\hline No income & $12(32.4)$ & $34(23.8)$ & & & & \\
\hline \multicolumn{7}{|l|}{ Source of domestic water } \\
\hline Public tap & $12(32.4)$ & $35(24.5)$ & \multirow{4}{*}{1.857} & \multirow{4}{*}{0.395} & \multirow{4}{*}{-} & \multirow{4}{*}{-} \\
\hline Private tap & $25(67.6)$ & $104(72.7)$ & & & & \\
\hline Dam & $0(0)$ & $1(0.7)$ & & & & \\
\hline River & $0(0)$ & $3(2.1)$ & & & & \\
\hline \multicolumn{7}{|l|}{ Water storage } \\
\hline Jerry cans & $24(64.9)$ & $80(55.9)$ & \multirow{5}{*}{0.959} & \multirow{5}{*}{0.327} & \multirow{5}{*}{-} & \multirow{5}{*}{-} \\
\hline Tank & $13(35.1)$ & $63(44.1)$ & & & & \\
\hline Sanitary facility & & & & & & \\
\hline Communal pit latrine & $15(40.5)$ & $42(29.4)$ & & & & \\
\hline Private pit latrine & $22(59.5)$ & $101(70.6)$ & & & & \\
\hline \multicolumn{7}{|l|}{ Caregiver handwashing practice } \\
\hline After visiting toilet & $13(35.1)$ & $41(28.7)$ & \multirow{3}{*}{1.985} & \multirow{3}{*}{0.371} & \multirow{3}{*}{-} & \multirow{3}{*}{-} \\
\hline Before preparing/cooking food & $2(5.4)$ & $19(13.3)$ & & & & \\
\hline Before meals & $22(59.5)$ & $83(58.0)$ & & & & \\
\hline With soap and water & $9(24.3)$ & $35(24.5)$ & \multirow{2}{*}{0.141} & \multirow{2}{*}{0.708} & \multirow[b]{2}{*}{-} & \multirow{2}{*}{-} \\
\hline With water only & $28(75.7)$ & $108(75.5)$ & & & & \\
\hline \multicolumn{7}{|l|}{ Healthcare-seeking facility } \\
\hline Private clinical/pharmacy & $3(8.1)$ & $17(11.9)$ & \multirow{2}{*}{0.425} & \multirow{2}{*}{0.514} & \multirow{2}{*}{-} & \\
\hline Public health facility & $34(91.9)$ & $126(88.1)$ & & & & - \\
\hline
\end{tabular}

** shows significance.

[17] but rarely reported elsewhere in the Central African Republic [18], Ethiopia, and Egypt [19]. In our study, the most prevalent serogroup was Shigella flexneri $(70 \%)$, a finding consistent with other reports from Nigeria [20], Central Africa Republic [18], Nepal [21], Iran, India, and Eritrea [22, 23]-(4). Epidemiologically, Shigella serogroups show geographical stratification based on the economic and development status of a country, as well as the virulence of a given serogroup. Contrary to the developed world, Shigella flexneri is most predominant in developing countries where people are likely to have acquired immunity to S. sonnei following frequent exposure to Plesiomonas shigelloides in faecally contaminated water [24].

The emergence and global spread of multidrug-resistant (MDR) strains continue to pose serious public health problems and complicate the selection of appropriate empirical therapy [25]. In this study, all the Shigella serogroups were $100 \%$ resistant to ampicillin (AMP), trimethoprimsulfamethoxazole (co-trimoxazole, SXT), and tetracycline (TE). To the best of our knowledge, this was the highest Shigella resistance level to these three antibiotics, not only in Somalia but also in the neighboring countries. Resistance ranging from $80 \%$ to $100 \%$ has been reported in Kenya [26], Central Africa Republic [18], and Iran [27, 28]. AMP, SXT, and TE are low-cost antibiotics available over-the-counter and often used as empiric therapy for diarrheal cases in many developing countries and, therefore, the observed high-level resistance may have arisen due to overuse or misuse of these drugs. Our finding suggests that AMP and SXT are no longer appropriate empiric therapy for the treatment of bacillary dysentery among children under five years in Banadir Hospital, Somalia.

The current World Health Organization (WHO) guidelines for the treatment of shigellosis recommends ciprofloxacin as a first-line option or either Pivmecillinam, ceftriaxone, or azithromycin (in adults only) as second-line antibiotics [2]. In this study, ciprofloxacin (CIP) was only effective against $S$. dysenteriae type 1 serogroup, but some strains of S. flexneri (19.2\%) and S. sonnei (50\%) remained resistant. These findings are inconsistent with Liu and colleagues report, documenting CIP resistance of $49.7 \%$ and $19.1 \%$ for S. flexneri and S. sonnei, respectively [29]. In the current study findings, $40 \%$ of the CIP-susceptible $S$. dysenteriae type 1 were ceftriaxone (CRO) resistant, and the highest CRO resistance occurred among $S$. sonnei (66.7\%) serogroup, followed by S. dysenteriae type 1 (40\%) and S. flexneri (38.5\%). The CRO resistance was higher than $33.8 \%$ for S. sonnei and lower than $45.7 \%$ for S. flexneri reported in China [29]. Our study findings suggest a diminishing clinical value of CIP and CRO in Somalia, posing serious public health concern due to drug-resistant Shigella infections.

In the current study, none of the serogroups was resistant to azithromycin (AZM), which corroborates with other findings elsewhere in Iran and the USA [27-30] but inconsistent with reports that documented decreasing susceptibility to AZM in India (48\%) [31], Palestine (42\%) [32], and Australia (13.1\%) [33]. This discrepancy may be due to differences in the composition of study populations, where the current study focused only on children under five years. 
WHO does not recommend AZM for bacillary dysentery in children under five years, which may account for the absence of resistance observed in the current study.

Based on resistance to two or more classes of antibiotics, we identified seven multidrug-resistant (MDR) phenotypes, dominated by AMP/SXT/TE (37/37, 100\%), followed by AMP/SXT/CRO or SXT/TE/CRO (6/37, 42.2\%), AMP/SXT/ TE/CRO (13/37, 35.1\%), AMP/SXT/CIP or SXT/TE/CIP (8/ 37, 21.6\%), AMP/SXT/TE/CIP (5/37, 13.6\%), and AMP/ SXT/TE/CIP/CRO (3/37, 8.1\%). MDR phenotypes of S. dysenteriae type 1 (AMP/SXT or AMP/TE or SXT/TE, and AMP/SXT/TE) were susceptible to ciprofloxacin, but $50 \%$ $(3 / 6)$ of $S$. sonnei isolates and S. flexneri $(19.5 \%, 5 / 26)$ remained resistant. These profiles are similar to those reported in Nepal, but the levels are higher in the current study [34]. Shigella MDR phenotypes are commonly associated with the carriage of plasmid-encoded drug resistance genes and horizontal plasmid transfer among clinical Shigella isolates ([35]. Additionally, the MDR Shigella emerge due to the AcrAB-TolC drug efflux pump, also involved in MDR among other Gram-negative bacteria such as Escherichia coli and Enterobacter aerogenes [36]. These suggest the mechanisms mediating drug resistance among the Shigella isolates recovered in children under five years in Banadir Hospital, Somalia.

4.1. Limitation. The findings may not represent the health situation for the entire Somali country because this was a single hospital-based study with a small sample size.

\section{Conclusions}

Our findings showed a high prevalence of shigellosis and Shigella flexneri as the predominant serogroup among children under five years of age in Banadir Hospital, Somalia. Ampicillin and trimethoprim-sulfamethoxazole are no longer appropriate treatments for shigellosis in children under five years in Banadir Hospital. MDR Shigella strains, including those resistant to ciprofloxacin and ceftriaxone, have emerged in Somalia, posing a public health challenge. Therefore, there is an urgent need for AMR surveillance and continuous monitoring to mitigate the further spread of the MDR Shigella strains in Banadir Hospital and beyond.

\section{Data Availability}

The primary data used to support the findings of this study are included within the article.

\section{Ethical Approval}

This study was approved by the Research and Ethics Committee of the Ministry of Health, Human Services, and Research in Somalia. Research clearance and the permit were granted by the Ministry of Culture and Higher Education. Permission to collect samples was obtained from Banadir Hospital management. Stool samples were collected where laboratory coding was used to identify the participants.
Information obtained from participants was treated with confidentiality and only used for the study.

\section{Consent}

Written informed consent was sought from patients or guardians of the research subjects before interviewing by first explaining the purpose of the study.

\section{Conflicts of Interest}

The authors declare no conflicts of interest.

\section{Authors' Contributions}

Nelson Chengo Menza and Abednego Moki Musyoki contributed equally to this work.

\section{Acknowledgments}

The authors want to express their gratitude to the management of Banadir Hospital for giving permission to carry out this study and the Ministry of Culture and Higher Education for granting research clearance and permit. Their acknowledgement goes to physicians and the pediatric department staff members of Banadir Hospital for their support during sample collection. The authors also thank staff members of the Somalia National Public Health Reference Laboratory (NPHRL) for providing laboratory facilities and the standard microorganism.

\section{References}

[1] K. L. Kotloff, J. P. Winickoff, B Ivanoff et al., "Global burden of Shigella infections: implications for vaccine development and implementation of control strategies," Bulletin of the World Health Organization, vol. 77, no. 8, pp. 651-666, 1999.

[2] P. C. M. Williams and J. A. Berkley, "Guidelines for the treatment of dysentery (shigellosis): a systematic review of the evidence," Paediatrics and International Child Health, vol. 38, no. 1, pp. S50-S65, 2018.

[3] D. J. Ingle, M. Easton, M. Valcanis et al., "Co-circulation of multidrug-resistant Shigella among men who have sex with men in Australia," Clinical Infectious Diseases, vol. 69, no. 9, pp. 1535-1544, 2019.

[4] D. G. Naik, "Prevalence and antimicrobial susceptibility patterns of shigella species in asmara," Journal of Microbiology, Immunology and Infection, vol. 39, no. 5, pp. 392-395, Eritrea, Northeast Africa, 2006.

[5] S. Harbarth, G. Kahlmeter, J. Kluytmans et al., Global Priority List of Antibiotic-Resistant Bacteria to Guide Research, Discovery, and Development of WHO, WHO, Geneva, Switzerland, 2017.

[6] A. H. Mokdad, I. Khalil, C. El Bcheraoui et al., "Burden of diarrhea in the eastern mediterranean region, 1990-2015: findings from the global burden of disease 2015 study," International Journal of Public Health, vol. 63, no. S1, pp. 109-121, 2018.

[7] UNICEF SOMALIA Annual Report, 2014, https://somalia.un. org/en/19194-unicef-somalia-annual-report-2014.

[8] R. M. Humphries and A. J. Linscott, "Practical guidance for clinical microbiology laboratories: diagnosis of bacterial 
gastroenteritis," Clinical Microbiology Reviews, vol. 28, no. 1, pp. 3-31, 2015.

[9] A. Cheryl, M. S. Bopp, A. Allen, M. D. Ries, and M. P. H. Joy G, M. S. Wells, Laboratory Methods for the Diagnosis of Epidemic Dysentery and Cholera Laboratory Methods for the Diagnosis of Epidemic Dysentery and Cholera, Centers for Disease Control and Prevention, Atlanta, GE, USA, 1999.

[10] WHO, Manual for the Laboratory Identification of Bacterial Pathogens of Public Health Importance in the Developing World, World Health Organization, Geneva, Switzerland, 2003, https://www.semanticscholar.org/paper/Manual-forthe-laboratory-identification-and-of-of-Perilla-Ajello/eabce 7 fff543e01206c8e81fd3ddbfa7aeaacd1b.

[11] Clinical and Laboratory Standards Institute (CLSI), M100Ed30| Performance Standards for Antimicrobial Susceptibility Testing, 30th Edition Performance Standards for Antimicrobial Susceptibility Testing, p. 332, Clinical and Laboratory Standards Institute (CLSI), Wayne, PA, USA, 2020, https://clsi.org/ standards/products/microbiology/documents/m100/.

[12] C. E. O’Reilly, P. Jaron, B. Ochieng et al., "Risk factors for death among children less than 5 years old hospitalized with diarrhea in rural Western Kenya, 2005-2007: a cohort study," PLoS Medicine, vol. 9, no. 7, pp. 2005-2007, 2012.

[13] A. G. Kahsay and Z. Teklemariam, "Prevalence of Shigella among diarrheic children under-5 years of age attending at Mekelle health center, north Ethiopia," BMC Research Notes, vol. 8, pp. 10-13, 2015.

[14] A. Assefa and M. Girma, "Prevalence and antimicrobial susceptibility patterns of Salmonella and Shigella isolates among children aged below five years with diarrhea attending Robe General Hospital and Goba Referral Hospital, South East Ethiopia," Tropical Diseases, Travel Medicine and Vaccines, vol. 5, no. 1, pp. 19-11, 2019.

[15] E. T. Rogawski Mcquade, F. Shaheen, F. Kabir, A. Rizvi, J. A. Platts-Mills, F. Aziz et al., "Epidemiology of shigella infections and diarrhea in the first two years of life using culture-independent diagnostics in 8 low-resource settings," PLoS Neglected Tropical Diseases, vol. 14, no. 8, pp. 1-17, 2020.

[16] M. G. Qayad, "Health care services in transitional Somalia," Bildhaan: An International Journal of Somali Studies, vol. 7, pp. 190-210, 2008.

[17] M. Anderson, P. J. Sansonetti, and B. S. Marteyn, "Shigella diversity and changing landscape: insights for the twenty-first century," Frontiers in Cellular and Infection Microbiology, vol. 6, pp. 45-49, 2016.

[18] S. Breurec, T. Frank, C. Rafaï et al., "Serotype distribution and antimicrobial resistance of shigella species in Bangui, Central African Republic, from 2002 to 2013," The American Journal of Tropical Medicine and Hygiene, vol. 99, no. 2, pp. 283-286, 2018.

[19] A. G. Kahsay and S. Muthupandian, "Research Article A review on Sero diversity and antimicrobial resistance patterns of Shigella species in Africa , Asia and South America," BMC Research Notes, vol. 9, pp. 1-6, 2016.

[20] A. Abdu, A. O. Aboderin, J. B. Elusiyan, D. O. Kolawole, and A. Lamikanra, "Serogroup distribution of Shigella in Ile-Ife, southwest Nigeria," Tropical Gastroenterology, vol. 34, no. 3, pp. 164-169, 2013.

[21] G. Wilson, J. M. Easow, C. Mukhopadhyay, and P. G. Shivananda, "Isolation \& antimicrobial susceptibility of Shigella from patients with acute gastroenteritis in Western Nepal," The Indian Journal of Medical Research, vol. 123, no. 2, pp. 145-150, 2006.
[22] M. I. Qureishi, A. Borji, M. Bokaeian et al., "Antimicrobial resistance of Shigella spp. isolated from diarrheal patients in Zahedan," Acta Medica Iranica, vol. 46, no. 5, pp. 413-416, 2008.

[23] M. Puzari, M. Sharma, and P. Chetia, "Emergence of antibiotic resistant Shigella species: a matter of concern," Journal of Infection and Public Health, vol. 11, no. 4, pp. 451-454, 2018.

[24] A. A. Shad and W. A. Shad, "Shigella sonnei: virulence and antibiotic resistance," Archives of Microbiology, vol. 203, no. 1, pp. 45-58, 2021.

[25] S. Zamanlou, M. A. Rezaee, M. Aghazadeh, R. Ghotaslou, H. H. Nave, and Y. Khalili, "Genotypic diversity of multidrug ResistantShigellaspecies from Iran," Infection \& Chemotherapy, vol. 50, no. 1, pp. 29-37, 2018.

[26] W. K. Sang, V. Oundo, and D. Schnabel, "Prevalence and antibiotic resistance of bacterial pathogens isolated from childhood diarrhoea in four provinces of Kenya," Journal of Infection in Developing Countries, vol. 6, no. 7, pp. 572-578, 2012.

[27] R. Nikfar, A. Shamsizadeh, M. Darbor, S. Khaghani, and M. Moghaddam, "A Study of prevalence of Shigella species and antimicrobial resistance patterns in paediatric medical center in Ahvaz," Iran, vol. 9, no. 5, pp. 277-282, 2017.

[28] E. Abbasi, H. Abtahi, A. van Belkum, and E. Ghaznavi-Rad, "Multidrug-resistant Shigella infection in pediatric patients with diarrhea from central Iran," Infection and Drug Resistance, vol. 12, pp. 1535-1544, 2019.

[29] Y. Liu, H. Li, N. Lv et al., "Prevalence of plasmid-mediated determinants with decreased susceptibility to azithromycin among Shigella isolates in anhui, China," Frontiers in Microbiology, vol. 11, Article ID 1181, 2020.

[30] N. Singh, "Treatment of drug-resistant Shigella infections," Expert Review of Anti-infective Therapy, vol. 13, no. 1, pp. 69-80, 2015.

[31] D. Bhattacharya, H. Bhattacharya, R. Thamizhmani et al., "Shigellosis in Bay of Bengal Islands, India: clinical and seasonal patterns, surveillance of antibiotic susceptibility patterns, and molecular characterization of multidrug-resistant Shigella strains isolated during a 6-year period from 2006 to 2011," European Journal of Clinical Microbiology \& Infectious Diseases, vol. 33, no. 2, pp. 157-170, 2014.

[32] M. Salah, I. Shtayeh, R. Ghneim et al., "Evaluation of shigella species azithromycin CLSI epidemiological cutoff values and macrolide resistance genes," Journal of Clinical Microbiology, vol. 57, no. 4, pp. 1-7, 2018.

[33] J. D. Brown, S. J. Willcox, N. Franklin et al., "Shigella species epidemiology and antimicrobial susceptibility: the implications of emerging azithromycin resistance for guiding treatment, guidelines and breakpoints," Journal of Antimicrobial Chemotherapy, vol. 72, no. 11, pp. 3181-3186, 2017.

[34] G. Shakya, J. Acharya, S. Adhikari, and N. Rijal, "Shigellosis in Nepal: 13 years review of nationwide surveillance," Journal of Health, Population and Nutrition, vol. 35, no. 1, 2016.

[35] I.-F. Huang, C.-H. Chiu, M.-H. Wang, C.-Y. Wu, K.-S. Hsieh, and C. C. Chiou, "Outbreak of dysentery associated with ceftriaxone-resistant Shigella sonnei: first report of plasmidmediated CMY-2-type AmpC $\beta$-lactamase resistance in S. Sonnei," Journal of Clinical Microbiology, vol. 43, no. 6, pp. 2608-2612, 2005.

[36] H. Yang, G. Duan, J. Zhu et al., "The AcrAB-TolC pump is involved in multidrug resistance in ClinicalShigella flexneriIsolates," Microbial Drug Resistance, vol. 14, no. 4, pp. $245-249,2008$. 